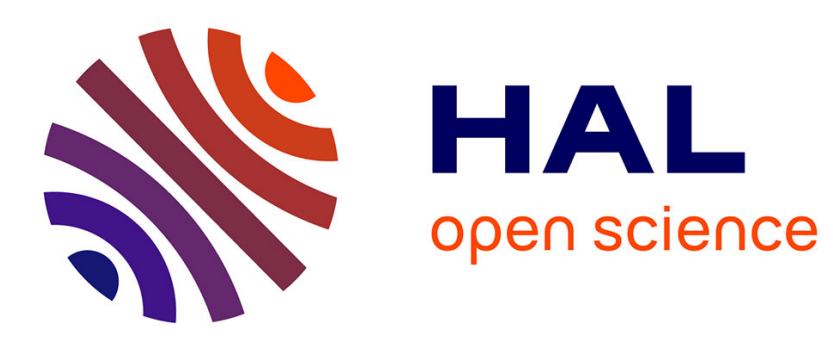

\title{
Heterogeneous Expectations, Exchange Rate Dynamics and Predictability
}

Sebastiano Manzan, Frank H. Westerhoff

\section{To cite this version:}

Sebastiano Manzan, Frank H. Westerhoff. Heterogeneous Expectations, Exchange Rate Dynamics and Predictability. Journal of Economic Behavior and Organization, 2007, 64 (1), pp.111. 10.1016/j.jebo.2006.08.005 . hal-00531865

\section{HAL Id: hal-00531865 https://hal.science/hal-00531865}

Submitted on 4 Nov 2010

HAL is a multi-disciplinary open access archive for the deposit and dissemination of scientific research documents, whether they are published or not. The documents may come from teaching and research institutions in France or abroad, or from public or private research centers.
L'archive ouverte pluridisciplinaire HAL, est destinée au dépôt et à la diffusion de documents scientifiques de niveau recherche, publiés ou non, émanant des établissements d'enseignement et de recherche français ou étrangers, des laboratoires publics ou privés. 


\section{Accepted Manuscript}

Title: Heterogeneous Expectations, Exchange Rate Dynamics and Predictability

Authors: Sebastiano Manzan, Frank H. Westerhoff

PII:

$$
\text { S0167-2681(07)00062-5 }
$$

DOI: doi:10.1016/j.jebo.2006.08.005

Reference: JEBO 2088

To appear in: Journal of Economic Behavior \& Organization

Received date:

Revised date:

Accepted date:
23-11-2004

22-7-2005

10-8-2006

Please cite this article as: Manzan, S., Westerhoff, F.H., Heterogeneous Expectations, Exchange Rate Dynamics and Predictability, Journal of Economic Behavior and Organization (2007), doi:10.1016/j.jebo.2006.08.005

This is a PDF file of an unedited manuscript that has been accepted for publication. As a service to our customers we are providing this early version of the manuscript. The manuscript will undergo copyediting, typesetting, and review of the resulting proof before it is published in its final form. Please note that during the production process errors may be discovered which could affect the content, and all legal disclaimers that apply to the journal pertain. 


\title{
Heterogeneous Expectations, Exchange Rate Dynamics and Predictability
}

\author{
Sebastiano Manzan ${ }^{\dagger *}$ and Frank H. Westerhoff ${ }^{\dagger}$ \\ $\dagger$ Department of Economics, University of Leicester \\ University Road, Leicester LE1 7RH, United Kingdom \\ phone: +44 116252 2854; fax: +44 1162522908 \\ e-mail: s.manzan@le.ac.uk \\ $\ddagger$ Department of Economics, University of Osnabrueck \\ Rolandstrasse 8, D-49069 Osnabrueck, Germany \\ phone: +49 541969 2743; fax: +49541969 12742 \\ e-mail: frank.westerhoff@uos.de
}

\begin{abstract}
This paper proposes a simple chartist-fundamentalist model in which we allow for nonlinear time variation in chartists' extrapolation rate. Estimation of the model using monthly data for the major currencies vis-a-vis the US dollar shows that the model is significant in-sample and that it has out-of-sample predictive power for some of the currencies. We investigate the power of tests of the random walk model to detect predictability against the alternative of the proposed model. We find that the evidence of short-term unpredictability and the long-term predictability are consistent with our model.

JEL code: F31, C52, C53.

Keywords: Exchange Rates, Heterogeneous Expectations, Forecasting, Nonlinear Models.
\end{abstract}

\footnotetext{
${ }^{*}$ Corresponding author.
} 


\section{Introduction}

The work of Meese and Rogoff (1983) showed that structural exchange rate models were not able to beat the simple random walk in out-of-sample prediction. Cheung et al. (2005) repeated a similar exercise, adding the last 20 years of data and confirming the earlier results of Meese and Rogoff. A possible reason for this failure is that fundamental variables used in the structural models play only a minor role in the determination of the exchange rate. This explanation was suggested in survey studies of exchange rate traders such as Frankel and Froot (1987), Allen and Taylor (1992), Lui and Mole (1998) and, more recently, Cheung and Chinn (2001). They find that traders perceive the effects of macroeconomic variables on exchange rates as a long-term phenomenon. For shorter horizons, their expectations are more influenced by non-fundamental factors, such as over-reaction to news, trading based on technical analysis and bandwagon effects. Hence, even though the structural models could explain the long-term dynamics of the exchange rate, at shorter horizons other (non-fundamental) factors play a relevant role in driving the exchange rate.

This evidence suggests that a successful model of exchange rate determination should combine both fundamental and non-fundamental factors. An early model along these lines is Frankel and Froot (1990). The model departs from rational expectations by assuming agents are of one of two types: fundamentalists or chartists. Fundamentalists form expectations about the exchange rate using fundamental factors, such as macroeconomic variables. Chartists, on the other hand, also use information about the past history of the exchange rate itself and extrapolate to form their expectations. The interaction of these types of traders is able to generate patterns with large deviations from the fundamentals. However, in the long term the stabilizing role of the fundamentalists' expectations drives the exchange rate back to its long-run equilibrium. Further interesting models with heterogeneous traders include Day and Huang (1990), Kirman (1991), De Grauwe and Dewachter (1993), De Grauwe and Grimaldi (2005), Brock and Hommes (1998), Lux and Marchesi (2000), Farmer and Joshi (2002) and Rosser et al. (2003). These models generate complex endogenous price dynamics and are able to match many important stylized facts of financial time series. For recent surveys of this topic see Hommes (2006) and LeBaron (2006). However, basically no attempt has been made thus far to estimate the interplay between chartists and fundamentalists, although this is a pressing matter given the high number of competing agent-based financial market models. Our paper seeks to make a first contribution in that direction.

In addition to the difficulty to explain the short-run dynamics by structural models, time 
series methods are also unable to make significant improvements in out-of-sample prediction. Diebold and Nason (1990) used nonparametric regression techniques on weekly exchange rates and failed to achieve any improvement. However, Mark (1995) found that the deviation of the nominal exchange rate from the monetary fundamental value has increasing explanatory power (both in- and out-of-sample) for multi-period returns as the horizon grows. More recently, the robustness of these results have been challenged by Kilian (1999) and Faust et al. (2003). They show that the evidence of predictability disappears when the sample period is extended and when data revisions are taken into account. The issue of (short- and long-horizon) predictability in exchange rates is still an open one. Engel and West (2005) offer an explanation for the inability to forecast nominal exchange rates using fundamental variables. In the set-up of a rational expectations present value model, they show that a large discount factor and a unit root in at least one forcing (observed or unobserved) variable imply a random walk behavior of the exchange rate. Another possible explanation for the poor forecasting performance of exchange rate models is provided by Kilian and Taylor (2003). They find evidence in favour of nonlinear dynamics in the deviation of the nominal exchange rate from the PPP fundamental value. Via simulation they show that long-horizon regression tests have high power in detecting predictability of multi-period returns but significantly lower power out-of-sample. They explain this fact as the effect of the small samples typically used for the out-of-sample exercise. Inoue and Kilian (2004) suggest that in-sample inference might be a more powerful tool to detect the (long-run) mean reversion behavior of asset prices towards their fundamental valuations.

In this paper, we propose an empirical model of exchange rate determination along the tradition of the chartist-fundamentalist approach. As in earlier models, we assume that market participants have heterogeneous expectations of the exchange rate: a group of traders (fundamentalists) considers fundamental factors while another group (chartists) regards also non-fundamental factors, such as past returns. We introduce a threshold type mechanism to model the time variation in the extrapolation rate of the chartists. We assume that they switch between two regimes according to the absolute change in the exchange rate being smaller or larger than a constant value. Further, chartists extrapolate more aggressively when the exchange rate increasingly deviates from the fundamentals. Estimation results suggest that there is evidence in support of the model for most of the currencies. In addition, the model is able to achieve statistically significant improvements in out-of-sample prediction for the Japanese Yen and the French Franc. This is an encouraging result given the evidence in the literature of short-horizon unpredictability.

In order to gauge the relevance of the proposed model to explain the stylized facts on the 
short-horizon and long-horizon predictability of spot exchange rates, we perform a Monte Carlo analysis. We simulate exchange rate series according to the proposed nonlinear model and series for the deviation from the PPP fundamental following a linear autoregressive model. We then forecast (out-of-sample) the simulated exchange rate series using the nonparametric method of Diebold and Nason and the long-horizon regression of Mark. By comparing these forecasts to those of the random walk model, we are able to evaluate the power of these methods to detect the short- and long-horizon predictability contained in the simulated series. In other words, the aim is to investigate the power of these tests to detect the predictability in the simulated spot exchange rate series due to the nonlinearity in the expectations of the chartists and the mean reversion due to the fundamentalist expectations. We find that the nonparametric test has extremely low power in detecting the predictability in the simulated series at the typical sample size available. On the other hand, the long-horizon test shows very high power in detecting the increasing in-sample predictability at longer horizons, while the power is significantly lower for the out-of-sample test. These results confirm the evidence and discussion presented in Kilian and Taylor. We interpret these results as suggestive that the failure to forecast exchange rates out-of-sample could be explained by the interaction of weak forms of nonlinearity and short samples typically used in forecasting.

The outline of the paper is as follows. In Section (2) we describe the model, and in Section (3) we show the estimation results for monthly exchange rates. In Section (4) we test whether the proposed structural model is actually consistent with the empirical puzzles previously mentioned. Finally, Section (5) concludes.

\section{The Model}

We develop a simple model of exchange rate determination in which investors have heterogeneous expectations. We assume the economy is populated by two types of traders. One group of traders, the fundamentalists, expects the exchange rate to converge to its long-run fundamental value. We assume their expectations are given by

$$
E_{t}^{f}\left(s_{t+1}\right)=s_{t}+\phi_{f}\left(f_{t}-s_{t}\right)
$$

where $s_{t}$ is the log of the exchange rate at time $t, f_{t}$ is the log of the fundamental value and $\phi_{f}$ is a parameter. We can rewrite this equation in terms of the expected change in the log 
exchange rate as

$$
E_{t}^{f}\left(r_{t+1}\right)=\phi_{f} z_{t}
$$

where $r_{t+1}=s_{t+1}-s_{t}$ is the one-period return and $z_{t}=f_{t}-s_{t}$ indicates the deviation of the fundamental value from the exchange rate. The fundamentalists expect tomorrow's price to include an adjustment component that corrects the mispricing of the exchange rate with respect to the fundamental value. In terms of returns, they expect the next period return to be proportional to the mispricing. A mean reversion behaviour of prices to the fundamentals implies that the coefficients $\phi_{f}$ should typically be positive and less than 1 . If the exchange rate at time $t$ is above (below) its long-run equilibrium, they expect a depreciation (appreciation) of $s_{t}$.

The second type of traders, the chartists, attribute a relevant role to the information extracted from the exchange rate itself. We assume that fundamentals are common knowledge to both groups, but chartists believe the information in the exchange rate itself carries information about its future dynamics (contrary to fundamentalists). We assume that the extrapolation rate of chartists depends on the magnitude of the deviation from the fundamentals. We model the expectations of this group as

$$
E_{t}^{c}\left(s_{t+1}\right)=s_{t}+\delta_{t}\left(s_{t}-s_{t-1}\right)
$$

where $\delta_{t}$ is a time-varying coefficient that captures the sentiment, or confidence, chartists have in the continuation or reversal of past returns. In terms of returns the expectation is

$$
E_{t}^{c}\left(r_{t+1}\right)=\delta_{t} r_{t}
$$

A positive value of $\delta_{t}$ is associated with bandwagon expectations in the sense that traders expect a trend to persist. If an appreciation (depreciation) of the exchange rate is observed they also expect a change in the same direction in the next period. On the other hand, if $\delta_{t}$ is negative they expect a reversal of the exchange rate in the following period. We interpret $\delta_{t}$ as indicating the sentiment that chartists have in the continuation of a trend. We assume that

$$
\delta_{t}= \begin{cases}\delta_{1}\left|z_{t}\right| & \text { if }\left|r_{t}\right| \geq c \\ \delta_{2}\left|z_{t}\right| & \text { otherwise }\end{cases}
$$

where the sentiment depends on the absolute deviation from the fundamentals and switches between two regimes, depending on the absolute return being bigger or smaller than a constant 
threshold value $c^{1}$. A long-standing discussion is the role of chartists' expectations in stabilizing or destabilizing the market. A negative $\delta_{t}$ is stabilizing in the sense that changes in the exchange rate are expected to reverse, whereas a positive $\delta_{t}$ is destabilizing in the sense that positive changes tend to persist and create trends in $s_{t}$. If the parameters $\delta_{1}$ and $\delta_{2}$ in Equation (3) have negative (positive) signs, then the chartists have a stabilizing (destabilizing) role. However, allowing $\delta_{t}$ to vary over time captures the fact that chartists might switch between destabilizing and stabilizing expectations. Opposite signs of the coefficients across regimes represents a situation in which they have stabilizing expectations in one regime and destabilizing ones in the other. This is an interesting hypothesis to test since it implies that chartists might contribute to correct or exacerbate deviations from the long-run equilibrium ${ }^{2}$. In addition, the $\delta_{t}$ captures the fact that chartists extrapolate more aggressively when the exchange rate deviates more from the fundamentals: if $\delta_{1}$ or $\delta_{2}$ are positive they become more confident about the continuation of the trend; on the other hand, if the parameters are negative they expect a stronger correction in the direction of the fundamentals.

We assume that investors have myopic mean-variance demand functions given by

$$
\begin{aligned}
& d_{t}^{f}=\alpha^{f}\left[E_{t}^{f}\left(s_{t+1}\right)-s_{t}\right] \\
& d_{t}^{c}=\alpha^{c}\left[E_{t}^{c}\left(s_{t+1}\right)-s_{t}\right],
\end{aligned}
$$

where the $\alpha$ 's are reaction coefficients and depend on the risk aversion coefficient. Finally, we assume that a risk-neutral market-maker aggregates the demands of the traders and adjusts the price according to the following rule

$$
s_{t+1}=s_{t}+\alpha^{m}\left[d_{t}^{f}+d_{t}^{c}\right]
$$

where $\alpha^{m}$ indicates the reaction coefficient of the market-maker.

\footnotetext{
${ }^{1}$ As in Day and Huang, the fundamental value is part of the information set of the chartists. Note also that models that allow for a switching between technical and fundamental trading rules implicitly assume that all traders are aware of the fundamentals.

${ }^{2}$ The stabilizing impact of the chartists may appear surprising since they are typically regarded as destabilizing positive feedback traders. However, Chiarella and He (2003) and He and Westerhoff (2005) analytically show that the behavior of the chartists may be beneficial for market stability in the presence of fundamentalists. Moreover, De Grauwe et al. (1993) explore the impact of technical trading rules on the dynamics of foreign exchange markets in more detail. In particular, they derive complex exchange rate dynamics when chartists adopt a trading strategy that uses both short-run and long-run moving averages into account. For instance, a technical sell signal may appear when a positive exchange rate trend loses its momentum (i.e. when the short-run exchange rate trend drops behind the long-run exchange rate trend). To clarify this aspect further, we do not restrict delta a priori.
} 


\section{Empirical Evidence}

We analyze monthly exchange rates from the beginning of 1974 to the end of 1998 . The currencies we consider are the German mark (DM), Japanese yen (JY), Canadian dollar (CD), French franc (FF) and the British pound (BP) against the US dollar. As the fundamental value we assume the PPP (Purchasing Power Parity) given by

$$
f_{t}=\pi_{t}-\pi_{t}^{*}
$$

where $\pi_{t}$ and $\pi_{t}^{*}$ indicate the log of the CPI index in the US and the foreign country, respectively. Figure (1) shows the time series and some linear properties of the exchange rates. From the first column of graphs it is immediately clear that there are large and persistent deviations of the nominal exchange rate from the PPP value for all currencies. The third column also highlights that nominal returns do not show any linear autocorrelation structure (the first 15 lags are displayed). Besides, the squared returns do not show significant linear dependence either.

\section{Figure (1)}

The model described in the previous section has a very simple structure that can be easily estimated. Setting all reaction coefficients equal (i.e. $\alpha^{f}=\alpha^{c}=\alpha^{m}=1$ ) implies the following model for returns:

$$
r_{t+1}=\delta_{1}\left|z_{t-r}\right| r_{t-p} I_{\left[\left|r_{t-q}\right| \geq c\right]}+\delta_{2}\left|z_{t-r}\right| r_{t-p} I_{\left[\left|r_{t-q}\right|<c\right]}+\phi_{f} z_{t-s}+\epsilon_{t+1}
$$

where $I_{(A)}$ denotes the indicator function that assumes the value 1 if $\mathrm{A}$ is true and zero otherwise, and $\epsilon_{t}$ is an i.i.d. observational noise term. In Equation (7) we generalize the model described in Section (2) to have lags ( $p, q, r$ and $s)$ in the expectations different from 0. We will search for the lags that best fit the data. Equation (7) is estimated by OLS because, conditional on the threshold $c$, the model is linear. We will perform a grid search for the optimal value of $c$.

We estimate the model in Equation (7) in the period 1974 to the end of 1994 and keep the observations from 1995 to the end of 1998 for one-step ahead out-of-sample forecasts. Table (1) reports the parameter estimates (with Newey-West standard errors) and the $F$-test for linearity proposed by Hansen $(1996,1997)^{3}$. To evaluate the out-of-sample forecasts we report the Root Mean Square Prediction Error (RMSPE) relative to the random walk model and the $\mathcal{D} \mathcal{M}$ test

\footnotetext{
${ }^{3}$ The p-values of the Hansen's $F$ statistic are obtained using 1000 simulations.
} 
proposed by Diebold and Mariano (1995) to compare predictive accuracy ${ }^{4}$. Table (1) shows also the estimation results for the linear model, that is, when we assume that $\delta_{1}=\delta_{2}=\delta_{0}$ are equal across regimes. In this case, we estimate the regression

$$
r_{t+1}=\delta_{0}\left|z_{t-r}\right| r_{t-p}+\phi_{f} z_{t-s}+\epsilon_{t+1}
$$

where $\delta_{0}$ is a parameter. We indicate Equation (8) as the linear model.

As shown in Table (1), for the linear model the lagged deviation from the fundamental price is significant at the $5 \%$ level for all the currencies, but the lagged value of the returns is not significant. Instead, when the nonlinear model is considered most of the coefficients are significant at the $5 \%$ level. The only exception is the CD, for which the evidence of a threshold dynamics is weak. This is also confirmed by the linearity test, which strongly rejects the null hypothesis of linearity for DM, FF and BP, rejects at the $10 \%$ level for the JY and does not reject for the $\mathrm{CD}^{5}$.

\section{Table (1)}

For most of the currencies the dependence of the return on the lagged deviation occurs at lag 12 (the $7^{\text {th }}$ lag is also significant for CD). The coefficient $\hat{\phi}_{f}$ varies between 0.018 for the JY and 0.037 for the BP, implying a slow adjustment towards the fundamentals. An interpretation of the results in terms of the model in Section (2) is that fundamentalists base their expectations about next month's exchange rate on the level of today's exchange rate with a typical $3 \%$ monthly adjustment of the deviation from the long-run equilibrium that occurred one year ago. This result is consistent with the survey analysis of Frankel and Froot (1987), Allen and Taylor (1992) and Cheung and Chinn (2001), from which it emerged that investors use information about fundamentals when forming long-term expectations.

The significant nonlinear dependence on past returns occurs on the first lag, with the exception of the BP, where the third lag is also significant. This confirms the survey findings

\footnotetext{
${ }^{4}$ We implement the $\mathcal{D} \mathcal{M}$ test with the correction proposed by Harvey et al. (1997). The p-value of the $\mathcal{D} \mathcal{M}$ is obtained using bootstrap methods under the null hypothesis of no exchange rate predictability.

${ }^{5}$ We followed the suggestion of a referee and considered the possibility that $\delta_{t}$ represents time variation in the sentiment of fundamentalists rather than chartists as we assume in our model. We estimate the alternative specification and the in-sample evidence for the nonlinear mechanism is very weak. The F-test statistic and p-values (in parenthesis) for the different currencies are DM 6.47 (0.08), JY 0.72 (0.98), CD 7.32 (0.07), FF $7.25(0.07)$ and $3.07(0.46)$ for the BP. The results indicate that for all currencies the nonlinear mechanism is not significant at 5\% significance level, although for three of them (DM, CD and FF) at 10\%. Comparing the test statistic with those reported in Table (1), it is clear that they are significantly smaller except in the case of the CD. In addition, the alternative specification does not outperform out-of-sample the random walk model for any of the currencies considered.
} 
previously cited, in which $90 \%$ of the respondents attributed a relevant role to technical analysis in the formation of expectations for short horizons. For all currencies, the sign of the estimated coefficients of $\delta_{t}$ are negative in the outer regime (for absolute returns higher than $c$ ) and positive in the inner regime (absolute returns smaller than $c$ ). This is evidence in support of the mixed influence of chartists' expectations on prices: when the exchange rate appreciation (depreciation) is smaller than the threshold, the chartists expect it to persist; on the other hand, when the observed change is larger than $c$, they expect a reversal of the change. Thus, there is evidence supporting the hypothesis that chartists act as a destabilizing force when absolute returns are small, but contribute to the stabilization of the exchange rate when they observe large movements in the exchange rate. In addition, the larger the deviation is from the fundamentals, the more aggressive they become in stabilizing (destabilizing). The estimated value of the threshold varies from $1.5 \%$ for the CD to a maximum of $3.2 \%$ for the DM.

To evaluate the role of this mechanism more effectively, we visualize the estimated dynamics of $\delta_{t}$ in Figure (2) for the case of the DM. In the top panel the coefficient $\delta_{t}$ is plotted against time; the bottom panel depicts the exchange rate and the PPP fundamental value. It is clear that $\delta_{t}$ is an indicator of chartist sentiment. In periods of large deviations they become nervous about the trading signals they extrapolate from the data. This fact is particularly clear in the period from 1980 to 1985, when a persistent appreciation of the US dollar occurred with respect to the DM that was not supported by an increase in the long-run equilibrium of the PPP. This period is associated with large variability in chartist sentiment, who reacted more nervously and aggressively to changes in prices as the deviation became larger. It is also interesting to note that in 1985 the extrapolation was so aggressive that chartists expected the next month's change to be even higher than the last observed change. We also observe that $\delta_{t}$ becomes negative in some cases. This confirms that they might have a stabilizing role when deviations are associated with large changes in the exchange rate (with respect to the threshold).

\section{Figure (2)}

The in-sample results are quite promising and point towards a significant explanatory power of the model. The out-of-sample prediction was carried out by re-estimating the model for each of the last 48 months of the sample and making a 1-step ahead forecast. The $\mathcal{D} \mathcal{M}$ statistics reported show significant evidence of predictability of the model for FF and JY at the $10 \%$ significance level. In the case of DM, JY and FF, the $\mathcal{D} \mathcal{M}$ statistic has a negative value, meaning that the structural model has a more accurate forecast ability than the random walk model. Hence, for 2 of the 6 currencies analyzed the in-sample evidence is confirmed even by 
the out-of-sample test. For DM and BP the out-of-sample results are not significant but the null of linearity can be safely rejected. For the CD there is evidence that the mean reversion to the fundamentals is stronger than for the other currencies.

\section{Evidence of Predictability?}

In the previous Section we concluded that the proposed model fits significantly well in-sample but that there is less robust evidence of significant out-of-sample predictive power. This result was already pointed out by Meese and Rogoff for linear models. Later, Diebold and Nason used nonparametric techniques to investigate nonlinear predictability for weekly exchange rates in a time series framework. They found significant in-sample improvements for a wide range of currencies, but out-of-sample the nonparametric method did not improve significantly over the random walk. They pointed to three possible reasons for the lack of out-of-sample improvements: (1) there might be dependence that occurs in even moments that cannot be exploited to improve predictions of the conditional mean, (2) evidence for in-sample nonlinearities could be spuriously caused by outliers and structural shifts, and (3) weak nonlinearities are present in the conditional mean, but it is difficult to exploit them in prediction at the typically available sample sizes. Further investigation of possible nonlinearities in the data was carried out by Meese and Rose (1991). They regressed nonparametrically the change in the exchange rate on some economic variables, such as interest rates, money growth rates and inflation rates. No significant improvement was found.

On the other hand, the unpredictability of exchange rate returns for short horizons has been challenged by evidence of predictability in long-horizon returns. Mark found evidence that the deviation of the nominal exchange rate from the monetary fundamental value has significant (insample and out-of-sample) explanatory power for multi-period returns. In addition, a typical finding is that the explanatory power increases with the horizons at which the returns are calculated. More recently, the findings of Mark have been questioned (see Kilian, 1999, and Faust et al., 2003). In particular, Faust et al. evaluate the robustness of Mark's findings of predictability to data revisions and sample period. Their results suggest that the evidence of predictability is limited to a sample period around that considered by Mark ${ }^{6}$, while extending the period and accounting for data revisions reverses the evidence of predictability. Kilian and Taylor find evidence of nonlinear mean reversion in the deviation of the nominal exchange rate from the PPP fundamental. Based on this evidence, they propose a modification of the

\footnotetext{
${ }^{6}$ Mark uses data from the first quarter of 1973 until the last quarter of 1991.
} 
bootstrap procedure used in long-horizon regressions (see Mark 1995, and Kilian 1999) that accounts for the nonlinear behavior of the real exchange rate. The application of this alternative test of the random walk hypothesis suggests strong evidence in support of increasing exchange rate predictability at longer horizons.

A heuristic analysis of Figure (1) suggests that the returns do not seem to be affected by the presence of outliers or heteroscedasticity. Thus, as Diebold and Nason pointed out, a likely explanation is that the interaction of weak nonlinearities and small samples is responsible for the lack of out-of-sample predictability for the exchange rates. The structural model proposed here could be used as a laboratory to investigate this issue. The model incorporates a nonlinearity, due to the switching mechanism in the chartists' expectations, that should be captured by nonparametric time series methods, but it also has a linear adjustment process to the longrun equilibrium, due to the fundamentalists' expectations. This second mechanism can be interpreted as the motivation behind the long-horizon predictability findings.

It is interesting, therefore, to generate time series from the structural model of Section (2) at the typical sample size available and forecast the simulated exchange rate series using the techniques of Diebold and Nason (1990) and Mark (1995). The aim is to evaluate whether the resulting (out-of-sample) forecasts are significantly more accurate compared to those of the random walk model. The comparison of prediction accuracy is based on the $\mathcal{D} \mathcal{M}$ test statistic proposed by Diebold and Mariano.

We simulate series for nominal exchange rates under the null hypothesis of no predictability and under the alternative hypothesis of predictability of the type proposed in Section (2). For the real exchange rate we assume it follows a linear autoregressive process ${ }^{7}$. Under the null hypothesis of no predictability we simulate exchange rate changes, $r_{t}$, and deviations from the PPP log-fundamental, $z_{t}=f_{t}-s_{t}$, as follows:

$$
\begin{gathered}
r_{t}=\mu_{r}+\epsilon_{t} \\
z_{t}=\mu_{z}+\sum_{j=1}^{p} \phi_{j} z_{t-j}+\eta_{t}
\end{gathered}
$$

where $\mu_{r}$ and $\mu_{z}$ are constants, and the noise terms $\epsilon_{t}$ and $\eta_{t}$ are uncorrelated and normally distributed with mean zero and variance equal to the estimated value for the two series. The lag order $p$ is chosen optimally according to the BIC criterion. The simulated exchange rate,

\footnotetext{
${ }^{7}$ Kilian and Taylor conduct a similar simulation exercise where they consider the alternative hypothesis of predictability deriving from a linear fundamental process and a nonlinear mean-reverting model for the real exchange rate.
} 
$s_{t}$, is obtained by cumulating the values of $r_{t}$. Under the alternative hypothesis of nonlinear predictability of the nominal exchange rate, we simulate series from the following model:

$$
\begin{gathered}
r_{t}=\mu_{r}+\left\{\delta_{1}\left|z_{t-1}\right| r_{t-1} I_{\left[\left|r_{t-1}\right| \geq c\right]}+\delta_{2}\left|z_{t-1}\right| r_{t-1} I_{\left[\left|r_{t-1}\right|<c\right]}+\phi_{f} z_{t-12}\right\}+\epsilon_{t} \\
z_{t}=\mu_{z}+\sum_{j=1}^{p} \phi_{j} z_{t-j}+\eta_{t}
\end{gathered}
$$

where for the parameter values $\phi_{1}, \phi_{2}, \phi_{f}$ and $\gamma$ we use the estimates in Section (3) for the different currencies. Based on the simulated series $r_{t}$ (or $s_{t}$ ) and $z_{t}$ in Equations (9) and (10), we apply the tests mentioned earlier to evaluate their size and power properties in detecting the predictability of the nominal exchange rate built into the model of Section (2). For the short-run predictability test we regress nonparametrically changes in the nominal exchange rate $r_{t}$ on its own lags, while for the long-horizon regression we regress the $k$-period change of the nominal exchange rate, $r_{t+k}=s_{t+k}-s_{t}$, on the deviation from the PPP fundamental $z_{t}$.

In Section (4.1) we present the nonparametric forecasting method used in Diebold and Nason, the out-of-sample predictability results for the five exchange rate series considered in the previous section, and the size and power analysis based on the simulation exercise described above. The same is reported in Section (4.2) for the long-horizon regression. However, in this case we perform both in- and out-of-sample analysis.

\subsection{Short-Horizon (Out-of-Sample) Unpredictability}

We use the locally weighted regression (LWR) framework of Cleveland and Devlin (1988). Previous applications of LWR to exchange rates are Diebold and Nason (1990) and Meese and Rose (1991). We assume the following nonparametric regression model for exchange rate returns:

$$
r_{t+1}=m\left(X_{t}\right)+\epsilon_{t}
$$

where $X_{t}$ is a vector of lagged values of $r_{t}$ and $\epsilon_{t}$ is an i.i.d. disturbance term. The LWR method estimates the conditional mean function at the point $x, m(x)$, by minimizing the following quantity:

$$
\sum_{t=1}^{n-1}\left\{r_{t+1}-\alpha-\beta\left(X_{t}-x\right)\right\}^{2} K\left(\frac{\left\|X_{t}-x\right\|}{d_{k}(x)}\right),
$$


where $\widehat{m}(x)=\hat{\alpha}$, and $K(\cdot)$ is the tricube kernel defined as

$$
K(u)=\left\{\begin{array}{cl}
\left(1-u^{3}\right)^{3} & \text { for } 0 \leq u<1 \\
0 & \text { otherwise }
\end{array}\right.
$$

$\|\cdot\|$ indicates the euclidean distance and

$$
d_{k}(x)= \begin{cases}\left\|X_{x(k)}-x\right\| & \text { for } 0<h \leq 1 \\ \left\|X_{x(n)}-x\right\| h^{\frac{1}{p}} & \text { for } h>1\end{cases}
$$

$X_{x(k)}$ denotes the $k$-th nearest neighbor of $x$, and $k$ is the integer part of $h n$. The bandwidth $h$ can be interpreted as the parameter that regulates the smoothness of the local linear fit. Given the evidence in the previous section that nonlinearities occur mainly in the first lag, we set $X_{t}=$ $r_{t-1}$. As in Section (3), we evaluate the out-of-sample prediction accuracy of the nonparametric regression with the $\mathcal{D} \mathcal{M}$ test statistic and the no-change forecast as the null model. The statistic captures the higher accuracy (if any) of the nonparametric prediction compared to the random walk forecast. A negative value of the test statistic means that the nonparametric method has a lower forecasting error than the no-change forecast. Asymptotically, the test statistic is standard normally distributed. However, given the limited sample period devoted to out-ofsample prediction, we rely on the bootstrap approach under the null of no predictability in the nominal exchange rate.

In Table (2) we present the results of the nonparametric one-step-ahead prediction of the nominal exchange rates for the same period (1995:1 to 1998:12) used in the previous section. We consider bandwidth values $h$ that vary from 0.1 to $1^{8}$. For all currencies (and all bandwidth) we cannot reject the null hypothesis that the predictions of the LWR method are equally accurate compared to the no-change forecast. For most currencies the p-values of the test are very large, with some exceptions such as the Japanese Yen (when the bandwidth is equal to 1) that has a p-value of 0.12. These results confirm the earlier findings of Diebold and Nason that, despite the significant evidence of nonlinearities in the dynamics of exchange rates, nonparametric methods are unable to outperform the random walk model in out-of-sample forecasts.

\section{Table (2)}

Table (3) offers an explanation for these findings. As discussed earlier, we simulate series

\footnotetext{
${ }^{8}$ In a genuine out-of-sample exercise we would have to choose the value of the bandwidth prior to generating the prediction. However, we chose to use different values for the bandwidth in order to evaluate the power performance of the nonparametric test across various bandwidth values.
} 
from the structural model with the parameter values estimated in the previous section and evaluate the ability of the LWR forecasts to outperform the random walk model. Table (3) reports the frequency of rejections at the $5 \%$ significance level of the null hypothesis of equal predictive accuracy in 1000 simulations. The first column shows the results for the series simulated under the null of no exchange rate predictability (the model in Equation 9). In this case we investigate the size of the test across the different bandwidth. The predictability test seems correctly sized with frequencies very close to the nominal significance level. However, the power of the test is remarkably low across the set of parameters estimated for the different currencies. The highest power is 0.304 that is obtained using the parameter values estimated for the British Pound. Across the currencies, for DM, FF and BP the test has power between $20 \%$ and $30 \%$, while for JY and CD the power is around 10\%. It is interesting to notice that the highest power of the test is obtained for the currencies that were found to have stronger evidence of nonlinearity. The p-values of the Hansen's F statistic in Table (1) for DM, FF, and $\mathrm{BP}$ are below $1 \%$, while for the other currencies they are higher than $5 \%$. This suggests that the nonparametric regression method is able to capture the nonlinearity in the simulated series. However, the statistical properties of the test (due to the limited samples used in out-of-sample forecasting) and/or the mildness of the nonlinearity in exchange rate dynamics result in poor power performance of the predictability test even when the underlying model is nonlinear.

\section{Table (3)}

This conclusion is consistent with the explanation of Diebold and Nason mentioned earlier on the interplay between moderate nonlinearities and sample size.

\subsection{Long-Horizon predictability}

As previously mentioned, Mark showed that long-horizon returns are consistently more predictable than short period returns. To investigate this property he proposed the following regression:

$$
r_{t+k}=\alpha_{k}+\beta_{k} z_{t}+\epsilon_{t+k}
$$

where the $k$-period return is given by $r_{t+k}=s_{t+k}-s_{t}$ and $z_{t}$ is the deviation of the fundamental value from the exchange rate. The random walk model implies that exchange rate changes are unpredictable based on current information (such as current and past $z_{t}$ or past changes) at short as well as long horizons. This hypothesis can be tested with the null hypothesis that $\beta_{k}$ is equal to 0 . However, a coefficient $\beta_{k}$ different from zero implies that the observed 
deviation has explanatory power for the returns over the following $k$ periods. In addition, the economically interesting alternative is $\beta_{k}>0$ : if the exchange rate is lower (higher) compared to the fundamental value $\left(z_{t}>0\right)$, then in the next $k$ periods the exchange rate will appreciate (depreciate). In other words, the exchange rate will mean revert to the long-run equilibrium in the next $k$ periods. Inference in this type of regressions is typically conducted using bootstrap methods as in Mark (1995) and Kilian (1999).

Mark found that by increasing $k$, the estimate of $\beta_{k}$ increases in magnitude and becomes significantly positive. In addition, the $R^{2}$ of the regression consistently increases, suggesting higher explanatory power at longer horizons. He also uses Equation (13) for an out-of-sample prediction exercise and found significant improvements in forecasting performance (compared to the random walk model) at long horizons. However, this evidence is not robust to changes in the sample period and/or data revision as shown by Faust et al.. These results are based on the monetary model of exchange rate determination. When the PPP model is considered to specify the fundamental value of the exchange rate, Kilian and Taylor showed that there is strong evidence of long-horizon predictability in nominal exchange rates.

Previous studies of the long-horizon predictability of the nominal exchange rate have mainly used quarterly data for the post-Bretton Woods period. Instead, we consider monthly observations. The in-sample and out-of-sample periods are given as 1974:1-1994:12 and 1995:1 to 1998:12, respectively. We consider horizons $k$ from 1 month up to 36 months for both the in-sample and out-of-sample results. Table (4) reports the estimation results for the exchange rate series used in the previous Section.

\section{Table (4)}

For most currencies (except the Japanese Yen) increasing the horizon $k$ from 1 to 36 months, the estimates of $\beta_{k}$ become increasingly positive and statistically significant at the $10 \%$ level. This evidence supports the results of Mark (1995) and Kilian and Taylor (2003) ${ }^{9}$ of significant in-sample predictability at long horizons. For the JY there is no evidence of long-horizon predictability using monthly observations. The column $\mathcal{D M}_{k}$ in Table (4) reports the results of the out-of-sample long-horizon forecasts. In this case, there is no evidence to reject the hypothesis of equal accuracy of the long-horizon regression compared to the random walk model forecasts. This result holds for the same currencies for which we found significant in-sample

\footnotetext{
${ }^{9}$ Our results are similar to Kilian and Taylor because we use a PPP fundamental rather than a monetary fundamental as in Mark (1995), Kilian (1999) and Faust et al. (2003).
} 
predictability. This confirms the earlier evidence on the difficulty to forecast nominal exchange rates out-of-sample even when we find in-sample evidence supporting the model.

Similar to the previous simulation exercise, we now evaluate the size and power properties of the in-sample and out-of-sample predictability tests based on the long-horizon regression. The first two columns of Table (5) show the rejection frequencies at the $5 \%$ significance level for the in-sample (denoted as $\beta_{k}$ ) and out-of-sample (denoted $\mathcal{D} \mathcal{M}_{k}$ ) tests at horizons from 1 up to 36 months. Under the null hypothesis of no exchange rate predictability, the frequencies are quite close to the nominal level for all horizons, indicating that both tests do not suffer from size distortion. The results of the power analysis show that the in-sample test has a significantly higher power to detect predictability compared to the out-of-sample test. The rejection frequencies for the in-sample test range from 0.67 to 0.93 while the maximum power of the out-of-sample test is 0.13 . In addition, the power of the in-sample test has the tendency to increase at medium horizons and slightly decrease at the longer ones.

\section{Table (5)}

These results suggest that the evidence of out-of-sample unpredictability of exchange rates might be explained by the negligible power properties of the tests used in the literature. This confirms also the conclusion of Kilian and Taylor (2003) and Inoue and Kilian (2004) that in-sample tests represent a more effective way to evaluate predictability in asset prices.

\section{Conclusion}

In this paper we proposed a simple model of the dynamics of exchange rates inspired by the chartist-fundamentalist approach. We found that combining dependence on fundamental variables and on lagged values of returns is supported by the data. The fundamentalists expect the exchange rate to adjust toward the long-run equilibrium with a typical correction of approximately $3 \%$ monthly on the deviation that occurred one year ago. In addition to the stabilizing influence of this group, chartists also contribute to correct the mispricing of the exchange rate: we found that when chartists observe an absolute change beyond a threshold (of approximately $2-3 \%$ ), they expect a reversal in the next period. On the other hand, for absolute returns smaller than the threshold, they believe that changes will persist. There is also evidence that chartists become more aggressive in extrapolating trends in exchange rates when the deviation from the long-run equilibrium is larger. 
However, the in-sample results are not supported by significant out-of-sample evidence of predictability. We find that our model provides more accurate forecasts for only two of the six currencies. We also consider the nonparametric tests of Diebold and Nason and the longhorizon regression of Mark, confirming the inability of these methods to detect (out-of-sample) predictability in the exchange rate series.

Motivated by these results, we perform a Monte Carlo analysis in which we generate series from the proposed structural model and apply these methods to evaluate their ability to capture the forecastability in the simulated exchange rate series. Our results suggest that these tests have very low power to identify the dependence of the type proposed in this paper. The explanation that emerges from this paper for the inability to uncover the predictability in exchange rates is the interaction of weak forms of nonlinearity and the small samples typically available in empirical research.

Finally, after Meese and Rogoff the out-of-sample performance of models has been the benchmark to judge the robustness of the findings of in-sample dependence in exchange rates. However, not much attention has been paid to the issue of the statistical properties of the tests used in evaluating the accuracy of out-of-sample predictions and the role of nonlinearities. A priority for future research is to develop inference methods that are robust in small and moderate samples.

\section{Acknowledgements}

We thank two anonymous referees and the Editor for detailed and helpful comments. 


\section{References}

Allen H., Taylor, M.P., 1992. The use of technical analysis in the foreign exchange market. Journal of International Money and Finance 11, 304-314.

Brock, W.A., Hommes C.H., 1998. Heterogeneous beliefs and routes to chaos in a simple asset pricing model. Journal of Economic Dynamics and Control 22, 1235-1274.

Cheung, Y.-W., Chinn, M.D., 2001. Currency traders and exchange rate dynamics: A survey of the U.S. market. Journal of International Money and Finance 20, 439-471.

Cheung, Y.-W., Chinn, M.D., Pascual, A., 2005. Empirical exchange rate models of the nineties: Are any fit to survive? Journal of International Money and Finance 24, 1150-1175.

Chiarella, C., He, X.-Z., 2003. Dynamics of beliefs and learning under $\mathbf{a}_{L}$ processes - the heterogeneous case. Journal of Economic Dynamics and Control 27, 503-531.

Cleveland, W.S., Devlin, S.J., 1988. Locally weighted regression: an approach to regression analysis by local fitting. Journal of the American Statistical Association 83, 596-610.

Day, R.H., Huang, W., 1990. Bulls, bears and market sheep. Journal of Economic Behavior and Organization 14, 299-329.

De Grauwe, P., Dewachter, H., 1993. A chaotic model of the exchange rate: The role of fundamentalists and chartists. Open Economies Review 4, 351-379.

De Grauwe, P., Dewachter, H., Embrechts, M., 1993. Exchange Rate Theory - Chaotic Models of Foreign Exchange Markets. Oxford: Blackwell.

De Grauwe, P., Grimaldi, M., 2005. The Exchange Rate and its Fundamentals in a Complex World. Review of International Economics 13, 549-575.

Diebold, F.X., Mariano, R.S., 1995. Comparing predictive accuracy. Journal of Business and Economic Statistics 13, 253-263.

Diebold, F.X., Nason, J.A., 1990. Nonparametric exchange rate prediction? Journal of International Economics 28, 315-332.

Engel, C., West, K.D., 2005. Exchange rates and fundamentals. Journal of Political Economy 113, $485-517$.

Farmer, J.D., Joshi, S., 2002. The price dynamics of common trading strategies. Journal of Economic Behavior and Organization 49, 149-171. 
Faust, J., Rogers, J.H., Wright, J.H., 2003. Exchange rate forecasting: the errors we've really made. Journal of International Economics 60, 35-59.

Frankel, J.A., Froot, K.A., 1987. Using survey data to test standard propositions regarding exchange rate expectations. American Economic Review 77, 133-153.

Frankel, J.A., Froot, K.A., 1990. Chartists, fundamentalists, and trading in the foreign exchange market. American Economic Review 80, 181-185.

Hansen, B., 1996. Inference when a nuisance parameter is not identified under the null hypothesis. Econometrica 64, 413-430.

Hansen, B., 1997. Inference in TAR models. Studies in Nonlinear Dynamics and Econometrics 1, $1-14$.

Harvey, D., Leybourne, S., Newbold, P., 1997. Testing the equality of prediction mean squared errors. International Journal of Forecasting 13, 281-291.

He, X.-Z., Westerhoff, F., 2005. Commodity markets, price limiters and speculative price dynamics. Journal of Economic Dynamics and Control 29, 1577-1596.

Hommes, C.H., 2006. Heterogeneous agent models in economics and finance. In: Judd, K.L., Tesfatsion, L. (Eds.). Handbook of Computational Economics, vol. II. Amsterdam:North-Holland, $1109-1186$.

Inoue, A., Kilian, L., 2004. In-sample and out-of-sample tests of predictability: which one should we use? Econometric Reviews 23, 371-402.

Kilian, L., 1999. Exchange rates and monetary fundamentals: what do we learn from long-horizon regressions? Journal of Applied Econometrics 14, 491-510.

Kilian, L., Taylor, M.P., 2003. Why is so difficult to beat the random walk forecast of exchange rates? Journal of International Economics 60, 85-107.

Kirman, A., 1991. Epidemics of opinion and speculative bubbles in financial markets. In: Taylor, M.P. (Ed.), Money and Financial Markets. Oxford: Blackwell, 354-368.

LeBaron, B., 2006. Agent-based computational finance. In: Judd, K.L., Tesfatsion, L. (Eds.). Handbook of Computational Economics, vol. II. Amsterdam:North-Holland, 1187-1234.

Lui, Y.-H., Mole, D., 1998. The use of fundamental and technical analysis by foreign exchange dealers: Hong Kong evidence. Journal of International Money and Finance 17, 535-545. 
Lux, T., Marchesi, M., 2000. Volatility clustering in financial markets: A micro-simulation of interacting agents. International Journal of Theoretical and Applied Finance 3, 675-702.

Mark, N.C., 1995. Exchange rates and fundamentals: Evidence on long-horizon predictability. American Economic Review 85, 201-218.

Meese, R.A., Rogoff, K., 1983. Empirical exchange rate models of the seventies: Do they fit out of sample? Journal of International Economics 14, 3-24.

Meese, R.A., Rose, A.K., 1991. An empirical assessment of non-linearities in models of exchange rate determination. Review of Economic Studies 58, 603-619.

Rosser, J.B., Jr., Ahmed, E., Hartmann, G.C., 2003. Volatility via social flaring. Journal of Economic Behavior and Organization 50, 77-87. 

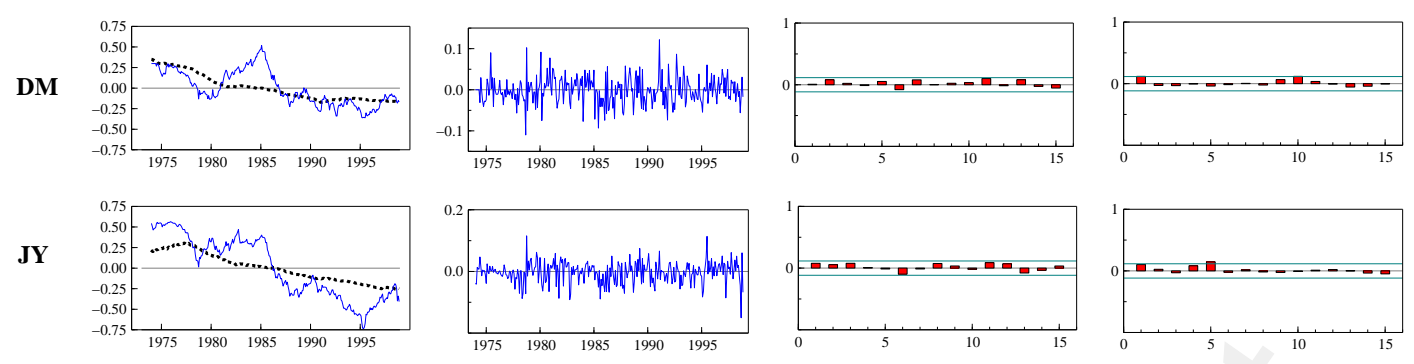

CD
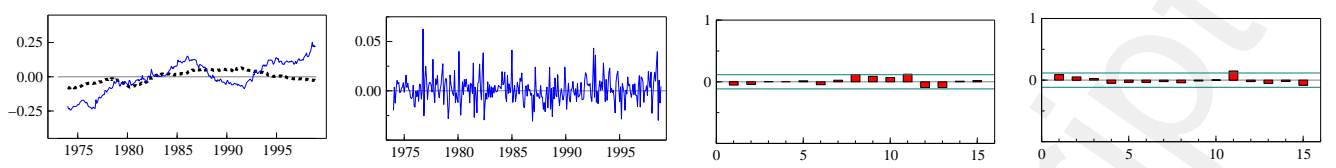

FF
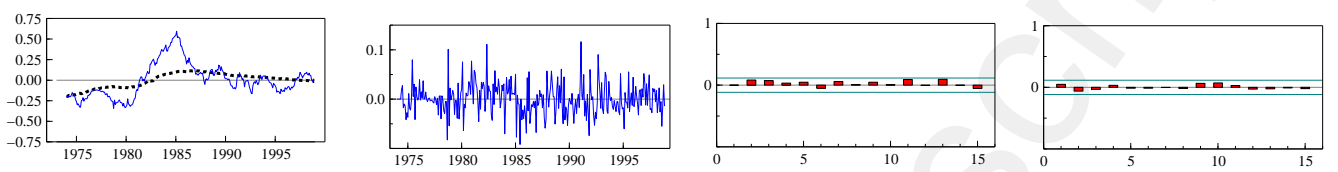

BP
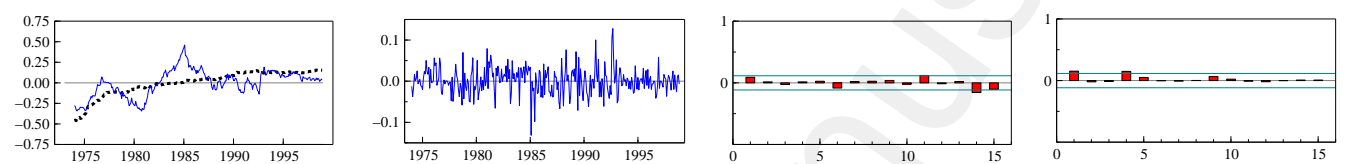

Figure 1: Exchange Rates: Plots for the major currencies against the US dollar for the sample period 1974-1998. The first column shows the nominal exchange rate and the PPP fundamental value, the second column shows the returns, the last two column are the ACF of the returns and the squared returns, respectively. 

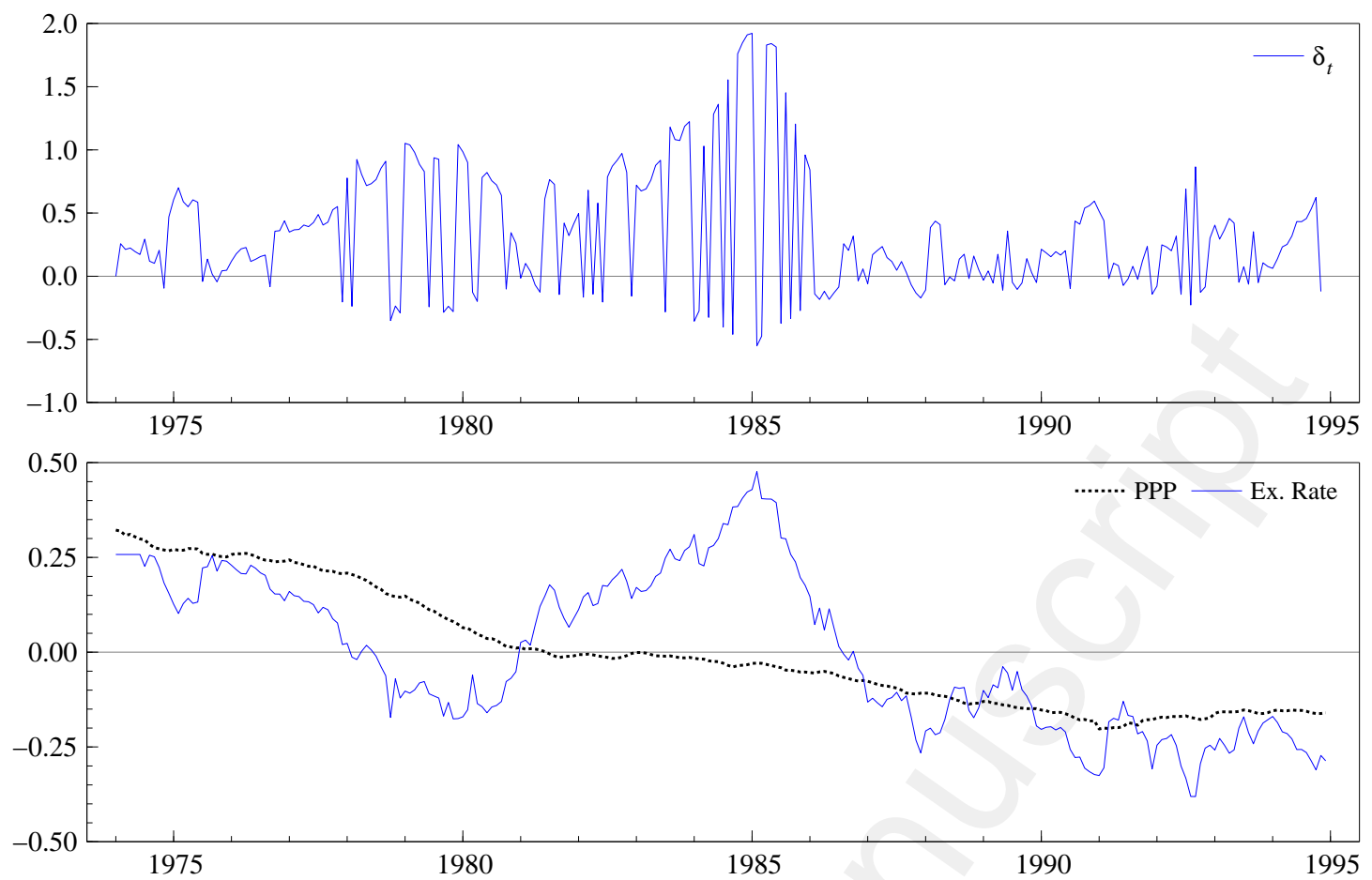

Figure 2: Chartists Extrapolation Coefficient: The evolution of $\delta_{t}$ for the DM exchange rate from 1974.1 to 1994.12 (top plot) and the log exchange rate and PPP fundamental value (bottom plot). 
Table 1: Estimation Results

\begin{tabular}{|c|c|c|c|c|c|c|c|c|c|c|}
\hline & & Linear & Model & & & & Jonline & Model & 80 & \\
\hline & & $\delta_{0}$ & $\phi_{f}$ & $\delta_{1}$ & $\delta_{2}$ & $c$ & $\phi_{f}$ & $F^{l i n}$ & RMSPE & $\mathcal{D} \mathcal{M}$ \\
\hline DM & $r_{t}$ & -0.047 & & -1.09 & 4.19 & 0.032 & & 29.44 & 0.963 & -0.89 \\
\hline & & $(-1.08)$ & & $(-2.59)$ & $(4.55)$ & & & $(0.00)$ & & $(0.18)$ \\
\hline & $z_{t-11}$ & & 0.027 & & & & 0.028 & & & \\
\hline & & & $(1.98)$ & & & & $(2.31)$ & 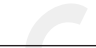 & $y$ & \\
\hline JY & $r_{t}$ & 0.38 & & -0.65 & 1.14 & 0.029 & & 7.43 & 0.961 & -1.33 \\
\hline & & $(1.22)$ & & $(-1.9)$ & $(2.75)$ & & & $(0.06)$ & & $(0.09)$ \\
\hline & $z_{t-11}$ & & 0.017 & & & & 0.018 & & & \\
\hline & & & $(1.81)$ & & & & $(2.35)$ & & & \\
\hline CD & $r_{t}$ & -0.73 & & -1.68 & 2.28 & 0.015 & & 3.89 & 1.04 & 0.38 \\
\hline & & $(-0.88)$ & & $(-2.01)$ & $(1.73)$ & & & $(0.44)$ & & $(0.65)$ \\
\hline & $z_{t-6}$ & & -0.07 & & & & -0.07 & & & \\
\hline & & & $(-2.6)$ & & & & $(-2.66)$ & & & \\
\hline & $z_{t-11}$ & & 0.09 & & & & 0.09 & & & \\
\hline & & & $(3.4)$ & & & & $(3.6)$ & & & \\
\hline FF & $r_{t}$ & -0.37 & & -0.84 & 3.89 & 0.029 & & 17.42 & 0.94 & -2.18 \\
\hline & & $(-0.87)$ & & $(-1.95)$ & $(3.78)$ & & & $(0.00)$ & & $(0.014)$ \\
\hline & $z_{t-11}$ & & 0.029 & & & & 0.031 & & & \\
\hline & & & $(2.06)$ & & & & $(2.31)$ & & & \\
\hline BP & $r_{t}$ & 0.50 & & & 3.78 & 0.025 & & 15.55 & 1.10 & 1.41 \\
\hline & & (1.00) & & & (3.1) & & & $(0.01)$ & & $(0.92)$ \\
\hline & $r_{t-2}$ & -0.34 & & -2.04 & & & & & & \\
\hline & & $(-0.72)$ & & $(-2.15)$ & & & & & & \\
\hline & $z_{t-11}$ & & 0.032 & & & & 0.037 & & & \\
\hline & & & $(2.11)$ & & & & $(2.41)$ & & & \\
\hline
\end{tabular}

Estimation results for the currencies in the first column vis-a-vis the U.S. dollar. The linear model is as in Equation (8) and the nonlinear model is given in Equation (7). The selected lags for the regressors are indicated in the second column. For the threshold variable the first lag was selected for all the currencies except for the JY where the threshold variable is $\left|r_{t-1}\right|$. The values in parentheses are the HCCE t-values. $F^{\text {lin }}$ indicates the F-test for linearity and in parentheses the simulated p-value; RMSPE indicates the root mean square (out-of-sample) prediction error for the last 48 observations; $\mathcal{D} \mathcal{M}$ indicates the statistic for the test of equality of the model forecast compared with the no-change forecast and the one-sided p-values in parentheses (a negative value of the statistics indicates that the structural model improves over the random walk model). Asymptotically it is standard normally distributed. We perform a one-sided test. 
Table 2: Nonparametric Predictability Test: applications to exchange rates

\begin{tabular}{|c|c|c|c|c|c|c|c|c|c|c|}
\hline \multirow[t]{2}{*}{ 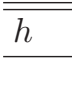 } & \multicolumn{2}{|c|}{ DM } & \multicolumn{2}{|c|}{ JY } & \multicolumn{2}{|c|}{$\overline{C D}$} & \multicolumn{2}{|c|}{ FF } & \multicolumn{2}{|c|}{ BP } \\
\hline & RMSPE & $\mathcal{D M}$ & RMSPE & $\mathcal{D M}$ & RMSPE & $\mathcal{D M}$ & RMSPE & $\mathcal{D M}$ & RMSPE & $\mathcal{D M}$ \\
\hline \multirow[t]{2}{*}{0.1} & 1.17 & 1.10 & 0.95 & -0.38 & 1.05 & 0.57 & 1.16 & 1.20 & 1.41 & 3.32 \\
\hline & & {$[0.68]$} & & {$[0.15]$} & & {$[0.49]$} & & {$[0.70]$} & & [1.00] \\
\hline \multirow[t]{2}{*}{0.2} & 0.98 & -0.22 & 0.92 & -0.70 & 0.99 & -0.09 & 1.04 & 0.40 & 1.25 & 2.75 \\
\hline & & {$[0.29]$} & & {$[0.14]$} & & {$[0.34]$} & & {$[0.52]$} & & {$[1.00]$} \\
\hline \multirow[t]{2}{*}{0.3} & 0.95 & -0.51 & 0.92 & -0.71 & 0.97 & -0.34 & 1.03 & 0.31 & 1.22 & 2.39 \\
\hline & & {$[0.25]$} & & {$[0.18]$} & & {$[0.29]$} & & {$[0.54]$} & & {$[1.00]$} \\
\hline \multirow[t]{2}{*}{0.4} & 0.94 & -0.59 & 0.92 & -0.74 & 0.97 & -0.47 & 1.01 & 0.14 & 1.18 & 2.32 \\
\hline & & {$[0.25]$} & & {$[0.20]$} & & {$[0.27]$} & & {$[0.49]$} & & {$[1.00]$} \\
\hline \multirow[t]{2}{*}{0.5} & 0.94 & -0.71 & 0.92 & -0.77 & 0.96 & -0.60 & 1.01 & 0.15 & 1.14 & 2.18 \\
\hline & & {$[0.20]$} & & {$[0.19]$} & & {$[0.24]$} & & {$[0.50]$} & & [1.00] \\
\hline \multirow[t]{2}{*}{0.6} & 0.93 & -0.80 & 0.92 & -0.79 & 0.96 & -0.66 & 1.00 & 0.08 & 1.12 & 2.09 \\
\hline & & {$[0.18]$} & & {$[0.20]$} & & {$[0.23]$} & & {$[0.48]$} & & {$[1.00]$} \\
\hline \multirow[t]{2}{*}{0.7} & 0.93 & -0.85 & 0.92 & -0.80 & 0.96 & -0.73 & 1.00 & 0.00 & 1.10 & 1.96 \\
\hline & & {$[0.17]$} & & {$[0.20]$} & & {$[0.20]$} & & {$[0.47]$} & & {$[0.99]$} \\
\hline \multirow[t]{2}{*}{0.8} & 0.93 & -0.89 & 0.91 & -0.85 & 0.95 & -0.79 & 0.99 & -0.08 & 1.08 & 1.80 \\
\hline & & {$[0.16]$} & & {$[0.18]$} & & {$[0.19]$} & & {$[0.45]$} & & {$[0.99]$} \\
\hline \multirow[t]{2}{*}{0.9} & 0.94 & -0.82 & 0.90 & -0.92 & 0.96 & -0.81 & 0.98 & -0.25 & 1.06 & 1.59 \\
\hline & & {$[0.20]$} & & {$[0.16]$} & & {$[0.19]$} & & {$[0.41]$} & & [0.99] \\
\hline \multirow[t]{2}{*}{1.0} & 0.98 & -0.34 & 0.89 & -0.99 & 0.98 & -0.66 & 0.98 & -0.36 & 1.05 & 1.22 \\
\hline & & {$[0.40]$} & & {$[0.12]$} & & {$[0.28]$} & & {$[0.39]$} & & [0.98] \\
\hline
\end{tabular}

Out-of-sample LWR forecast for the period 1995:1 to 1998:12. RMSPE is the root mean squared prediction error (relative to the random walk model), and $\mathcal{D} \mathcal{M}$ statistic tests the null hypothesis of equal prediction accuracy of the nonparametric and random walk forecasts. In square brackets are the bootstrap p-values under the null hypothesis of no predictability in the exchange rate. 
Table 3: Size and Power of the Nonparametric Predictability Test

\begin{tabular}{lcccccc}
\hline \hline$h$ & null & DM & JY & CD & FF & BP \\
\hline 0.1 & 0.051 & 0.194 & 0.063 & 0.090 & 0.147 & 0.215 \\
0.2 & 0.049 & 0.216 & 0.066 & 0.114 & 0.165 & 0.246 \\
0.3 & 0.042 & 0.237 & 0.088 & 0.116 & 0.193 & 0.258 \\
0.4 & 0.040 & 0.247 & 0.088 & 0.124 & 0.194 & 0.265 \\
0.5 & 0.034 & 0.251 & 0.096 & 0.128 & 0.204 & 0.285 \\
0.6 & 0.037 & 0.262 & 0.101 & 0.125 & 0.201 & 0.290 \\
0.7 & 0.044 & 0.268 & 0.098 & 0.119 & 0.211 & 0.300 \\
0.8 & 0.046 & 0.262 & 0.108 & 0.116 & 0.197 & 0.304 \\
0.9 & 0.052 & 0.258 & 0.118 & 0.112 & 0.177 & 0.299 \\
1.0 & 0.048 & 0.180 & 0.133 & 0.072 & 0.072 & 0.282 \\
\hline \hline
\end{tabular}

Frequency of rejection of the null hypothesis of equal predictive accuracy of the nonparametric and random walk out-of-sample forecasts. The null represents the model in Equation (9) while the alternative investigated here is the model in Equation (10). The simulated time series are of length 300, of which the last 48 are predicted out-of-sample. The test is the $\mathcal{D} \mathcal{M}$ test for prediction accuracy. The entries indicate the power of the test (i.e. the frequency of rejection of the null hypothesis of equal accuracy of the LWR and no-change predictions when the time series are simulated from the structural model at the estimated parameter values of the different currencies). 
Table 4: Long-Horizon Predictability Tests: Applications to exchange rates

\begin{tabular}{|c|c|c|c|c|c|c|c|c|c|c|}
\hline \multirow[t]{2}{*}{$k$} & \multicolumn{2}{|c|}{ DM } & \multicolumn{2}{|c|}{ JY } & \multicolumn{2}{|c|}{$\mathrm{CD}$} & \multicolumn{2}{|c|}{ FF } & \multicolumn{2}{|c|}{$\mathrm{BP}$} \\
\hline & $\beta_{k}$ & $\mathcal{D} \mathcal{M}_{k}$ & $\beta_{k}$ & $\mathcal{D} \mathcal{M}_{k}$ & $\beta_{k}$ & $\mathcal{D} \mathcal{M}_{k}$ & $\beta_{k}$ & $\mathcal{D} \mathcal{M}_{k}$ & $\beta_{k}$ & $\mathcal{D} \mathcal{M}_{k}$ \\
\hline \multirow[t]{2}{*}{1} & 0.015 & -0.919 & 0.005 & -1.631 & 0.007 & 0.677 & 0.016 & -0.63 & 0.023 & 0.60 \\
\hline & {$[0.15]$} & {$[0.17]$} & {$[0.31]$} & {$[0.051]$} & {$[0.22]$} & {$[0.66]$} & {$[0.16]$} & {$[0.23]$} & {$[0.13]$} & {$[0.63]$} \\
\hline \multirow[t]{2}{*}{12} & 0.225 & -1.052 & 0.066 & -1.545 & 0.153 & 0.955 & 0.258 & -0.57 & 0.326 & 2.023 \\
\hline & {$[0.19]$} & {$[0.18]$} & {$[0.34]$} & {$[0.097]$} & {$[0.14]$} & {$[0.69]$} & {$[0.18]$} & {$[0.29]$} & {$[0.04]$} & {$[0.92]$} \\
\hline \multirow[t]{2}{*}{24} & 0.529 & -0.722 & 0.224 & -0.901 & 0.466 & 0.865 & 0.618 & -0.411 & 0.639 & 1.359 \\
\hline & {$[0.09]$} & {$[0.23]$} & {$[0.25]$} & {$[0.19]$} & {$[0.078]$} & {$[0.68]$} & {$[0.045]$} & {$[0.31]$} & {$[0.01]$} & {$[0.83]$} \\
\hline \multirow[t]{2}{*}{36} & 0.814 & 0.345 & 0.0 .462 & -0.352 & 0.762 & 0.399 & 1.047 & 0.205 & 1.024 & 1.168 \\
\hline & {$[0.014]$} & {$[0.28]$} & {$[0.18]$} & {$[0.30]$} & {$[0.084]$} & {$[0.61]$} & {$[0.00]$} & {$[0.52]$} & {$[0.03]$} & {$[0.89]$} \\
\hline
\end{tabular}

Estimation and forecasting results of the long-horizon regression in Equation (13) for the five exchange rate series considered. $k$ (from 1 to 36 months) denotes the period for which the long-horizon return is calculated. The sample period for estimation is from 1974:1 to 1994:12. The out-of-sample forecasts are generated recursively for the period 1995:1 up to 1998:12. The null hypothesis is that $\beta_{k}=0$ versus the one-sided alternative that is larger than 0 . The $\mathcal{D} \mathcal{M}$ statistic tests the null hypothesis of equal accuracy of the nonparametric and the random walk model forecasts. The values reported in square brackets are the one-sided p-values obtained using the bootstrap method in Mark (1995). In bold are the p-values below $10 \%$. 
Table 5: Size and Power of the Long-Horizon Predictability Tests

\begin{tabular}{|c|c|c|c|c|c|c|c|c|c|c|c|c|}
\hline \multirow[t]{2}{*}{$k$} & \multicolumn{2}{|c|}{ null } & \multicolumn{2}{|c|}{ DM } & \multicolumn{2}{|c|}{ JY } & \multicolumn{2}{|c|}{ CD } & \multicolumn{2}{|c|}{ FF } & \multicolumn{2}{|c|}{ BP } \\
\hline & $\beta_{k}$ & $\mathcal{D} M$ & $\beta_{k}$ & $\mathcal{D} M$ & $\beta_{k}$ & $\mathcal{D} M$ & $\beta_{k}$ & $\mathcal{D} M$ & $\beta_{k}$ & $\mathcal{D} M$ & $\beta_{k}$ & $\mathcal{D} M$ \\
\hline 1 & 0.049 & 0.053 & 0.670 & 0.111 & 0.663 & 0.092 & 0.737 & 0.134 & 0.714 & 0.10 & 0.895 & 0.103 \\
\hline 12 & 0.056 & 0.054 & 0.809 & 0.111 & 0.693 & 0.110 & 0.854 & 0.106 & 0.848 & 0.115 & 0.938 & 0.124 \\
\hline 24 & 0.046 & 0.058 & 0.843 & 0.113 & 0.705 & 0.091 & 0.844 & 0.09 & 0.894 & 0.098 & 0.926 & 0.111 \\
\hline 36 & 0.050 & 0.056 & 0.826 & 0.095 & 0.695 & 0.071 & 0.773 & 0.096 & 0.851 & 0.097 & 0.872 & 0.097 \\
\hline
\end{tabular}

Frequency of rejection of the in-sample and out-of-sample test of no nominal exchange rate predictability. The in-sample test consists of the null hypothesis that $\beta_{k}=0$ (versus the onesided alternative that is larger than 0 ). The null hypothesis of the out-of-sample test is the equal accuracy of the long-horizon and random walk forecasts. The exchange rate and deviation from the PPP fundamental series are generated according to model (9) (under the null of no exchange rate predictability) and (10) (under the alternative). In this case, we use the parameters reported in Table (1) for the different currencies. 